\title{
Impact of Modification Process on Graphite Structure at Application of Chloroperfluorododecylfluoro Sulfate
}

\author{
Marina Gurgenishvili*, Givi Papava, Vitali Sherozia, Ia Chitrekashvili, Rusudan Tsiskarishvili and Irma \\ Jinikashvili \\ Petre Melikishvili Institute of Physical and Organic Chemistry, Ivane. Javakhishvili Tbilisi State University, Tbilisi 0179, Georgia
}

\begin{abstract}
Impact of the process of modification on graphite structure at the application of fluorine-containing oligomer-FDS (chloroperfluorododecylfluoro sulfate) has been studied. It was shown that during this process graphite structure does not change. Only particles of FDS are destructed into monolayers, which are spread in graphite intercrystalline voids. At the mechanical impact it protects graphite from destruction and correspondingly, improveing some of its tribotechnical characteristics.
\end{abstract}

Key words: Grafit, modification, diffractometer, FDS, oligomer.

\section{Introduction}

Graphite, thanks to its unique properties, it is widely used in various spheres of human activity, including production of antifriction materials [1, 2]. In solid antifriction materials it can be used as a matrix, as well as additive, in plastic and liquid lubricants-admix, and in polymer self-lubricating antifriction materials - as filler. Exploitation properties of these materials depend significantly on the properties of graphite. Therefore, graphite is often subjected to modification. Selection of modified graphite for any definite material is made according to definite technical demands. Therefore, widening of nomenclature of such modified graphite is a rather urgent task.

The present paper deals with graphite modification by the use of fluorine-containing oligomer-FDS (chloroperfluorododecylfluoro sulfate). As it turned out, lubricating properties of such graphite are improved markedly. This conditioned our interest to study the effect of this process on graphite structure, in order to determine the mechanism of improvement of antifriction properties.

\footnotetext{
*Corresponding author: Marina Gurgenishvili, Ph.D/chief scientific researcher, research fields: anti-friction composite materials, ionits, thermo-reactive polymers, plating and prolongation.
}

\section{Materials and Methods}

Initial materials were natural crystalline graphite and fluorine-containing oligomer-FDS, obtained from 1-hydro-12-chloroperfluorododecane via treatment by peroxide sulphuryl di-fluorine in conditions analogous to those described earlier for 1-hydro perfluorine alkenes [3].

$$
\mathrm{Cl}\left(\mathrm{CF}_{2}\right)_{12} \mathrm{H}+\mathrm{S}_{2} \mathrm{O}_{6} \mathrm{~F}_{2} \underset{-\mathrm{HSO}_{2} \mathrm{~F}}{\longrightarrow} \mathrm{Cl}\left(\mathrm{CF}_{2}\right)_{12} \mathrm{OSO}_{2} \mathrm{~F}
$$

Samples of modified graphite were prepared by joint treatment of graphite and FDS mixture in vibrating mill or by friction between two steel discs at $P=1 \mathrm{~kg} / \mathrm{cm}$ charge and $V=0.35 \mathrm{~m} / \mathrm{s}$ rate for $4 \mathrm{~min}$. Lubricating properties of the initial and modified graphite were estimated by their testing between two fractionating solid surfaces, at the terms of speed increase by definite intervals. Speed limit value corresponded to instantaneous growth of friction coefficient.

Investigation of the initial specimens and those obtained by their treatment was performed by X-ray method on a diffractometer "DRON-1", at $\mathrm{CuK} \alpha$ emission terms. Samples were subjected to XRD (X-ray diffraction) roentgenography in isotropic state in the form of powder or paste. To receive XRD patterns of the product obtained by friction, metal 
sample together with the layer rubbed on it, was placed in diffractometer. At the reflection analysis the authors obtained diffraction patterns of graphite-FDS mix surface layer.

\section{Results and Analysis}

Let's consider the structure of initial materials first graphite structure is known [3]. The main diffraction maximum (002) of graphite $2 \theta=26.6^{\circ}\left(d_{002}=3.35 \AA\right)$. Fig. 1 offers diffraction pattern of FDS, on which ciphers 2, 3, 4, 5 and 6 refer to the order of reflection, corresponding to the main reflex $d_{1}=17.80 \AA$. The authors also have the reflex $d_{2}=4.90 \AA$ and wide maximum in the region $2 \theta$ from $32^{\circ}$ to $44^{\circ}$. Diffraction picture obtained from FDS is analogous to diffraction obtained from paraffin and other substances possessing laminated structure. Period $d_{1}=17.80 \AA$ is inter-laminar period and it characterizes thickness of one layer. System of parallel layers is well ordered and extended at significant distance. This is proved by great quantity of orders of reflection from the main period $d_{1}$. Six reflection orders are seldom observed even in highly regulated laminated structures. Reflex, which corresponds to $2 \theta=18.15^{\circ}\left(d_{2}=4.90 \AA\right)$ is not inter-laminar, it is intra-laminar. Inter-planar spacing $d_{2}=4.90 \AA$ and wide maximums $32^{\circ}-44^{\circ}$ characterize inter molecular spacings in the layer. In diffraction pattern of PTFE (polytetrafluoroethylene), the high-molecular analogue of FDS, the main maximum also corresponds to $d_{2}=4.90 \AA$, and the remaining two reflexes are at $2 \theta 37.3^{\circ}$ and $41.5^{\circ}$. Intra-laminar maximums of FDS correspond to reflexes of PTFE, but maximums $37.3^{\circ}$ and $41.5^{\circ}$ are not resolved. Thus, it can be stated that molecules in the layer of FDS, are positioned approximately, as in the structure of PTFE, but with lower degree of order.

Since molecule axes in PTFE structure are perpendicular to elemental cell plane, molecular axes in FDS structure should be perpendicular to layer plane. Fig. 2 gives a scheme of structure of one FDS layer and a scheme of FDS crystalline, consisting of parallel layers. Molecules in the layer are positioned in approximately hexagonal lattice with inter molecular space $5.6 \AA$. Length of all molecules is strictly constant and equals to $17.80 \AA$. It proves that FDS molecules are mono-dispersive and have similar molecular masses. In PTFE structure $13 \mathrm{CF}_{2}$ [4] groups coincide with a period $16.9 \AA$ A. One group along the chain axis coincides with a segment $1.3 \AA$. Conformation of end groups $\mathrm{OSO}_{2} \mathrm{H}$ is not known precisely. The authors assume approximately that $\mathrm{CF}_{2}-\mathrm{O}-\mathrm{SO}_{2} \mathrm{~F}$ length along the chain axis equals to 4.5 $\AA$ [5]. Then $17.8-4.5=14.3 \AA$ and $14.3 / 1.3=11$, which corresponds to 12 groups of $\mathrm{CF}_{2}$. Thus, FDS

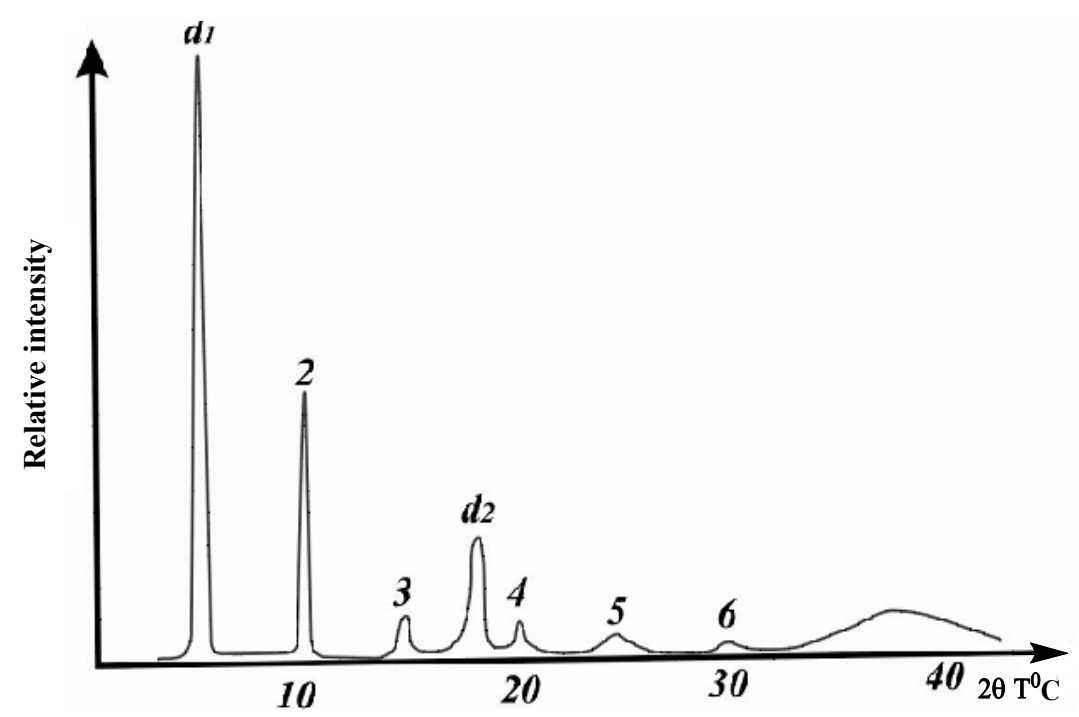

Fig. 1 Diffraction patterns of FDS. 


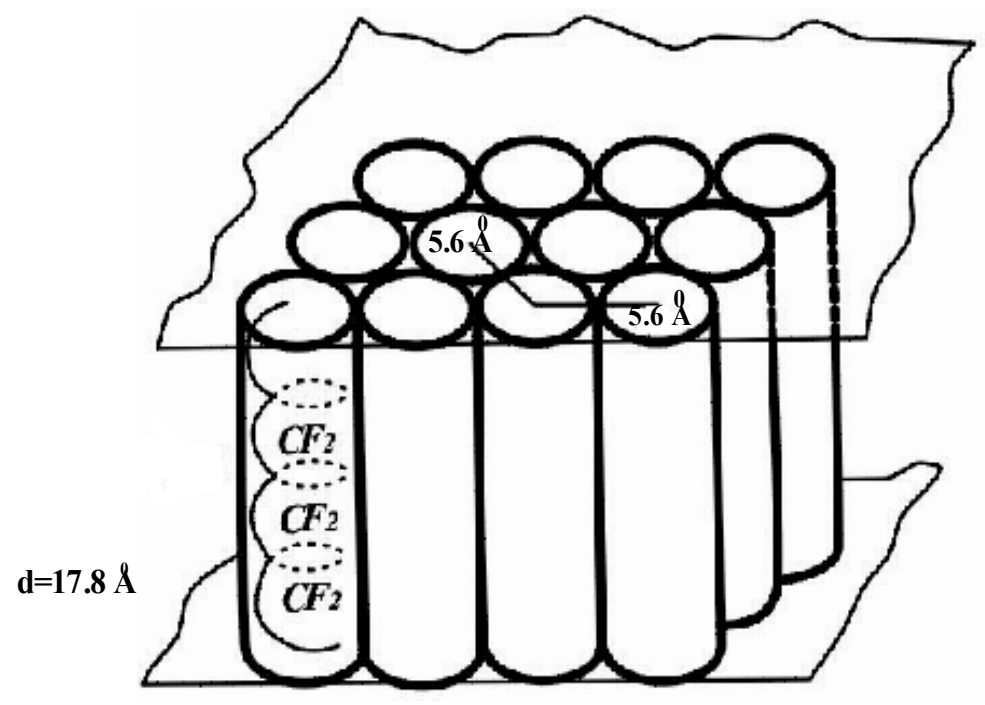

(a)

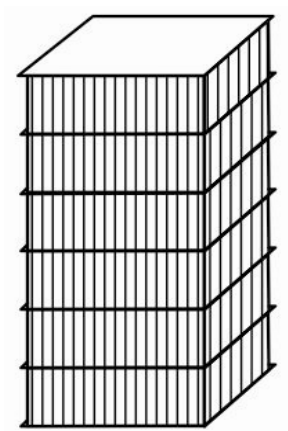

(b)

Fig. 2 Scheme of structure of one FDS layer (a) and of a crystallite consisting of parallel layers (b).

molecules in one layer are packed in hexagonal lattice with inter-chain spacing $5.6 \AA$. Molecule length or thickness of a layer is $17.80 \AA$. In the initial FDS layers are parallel and are packed in a crystallite, consisting of many layers.

Now let's consider graphite-FDS mix with FDS concentration $1 \%, 5 \%, 10 \%$ and $15 \%$. After crushing of graphite-FDS mix in a mortar structure of the components practically remain the same. Diffraction pattern of a mix after it is treated in a mortar is a sum of diffraction patterns of components with unaltered initial structure. Fig. 3 offers the main reflex of graphite (002) and all above listed reflexes of FDS. Further treatment of mixes was performed in vibrating mill and by friction between flat counter bodies. Structural changes at such treatments turned out similar, but rubbed layers on the counter bodies because of variable thicknesses are not good samples for diffractometer. This is why in the Fig. 3 we give diffraction patterns of samples prior (Fig. 3a) and after (Fig. 3b) treatment in vibrating mill. Comparison of diffraction patterns shows that as a result of treatment graphite line (002) suffers insignificant change. It becomes somewhat wider, which refers to definite decrease of graphite particle sizes after mechanical impact [6]. At analogous treatment of pure graphite in vibrating mill we observe greater widening of a line. In the initial graphite dimensions along the axis are minimum, $0.5-1 \mu \mathrm{m}$ and after treatment in a mill, they are reduced up to $150 \AA$. At the treatment of a mixture by $1 \%$ FDS, graphite crystallite sizes along the axis equal to $400 \AA$, and by 5\% FDS- $800 \AA$. The bigger the size of graphite crystallites, the lesser they are destructed at friction in vibrating mill. Thus, introduction of FDS into graphite protects graphite crystallites from destruction. As to the lines attributed to FDS, the main change after treatment in vibrating mill is that all orders of reflection connected with the inter-laminar period $d_{1}=17.80 \AA$ disappear. Simultaneously intra-laminar reflex $d_{2}=4.90 \AA$ is preserved and is clearly observable on diffraction patterns (Fig. 3b). Disappearance of inter-laminar reflex implies that the layers as such (or at least its significant part) are preserved, but the layer packages are destructed. After treatment of mixes in vibration mill FDS remains not in the form of particles, consisting of packages of layers, but in the form of separate mono layers. It is natural to suppose that such changes of structure are associated with the fact that separate FDS mono layers are spread and "smeared" at the borders of graphite particles. Structure of a mix after its treatment in vibrating mill can be imagines in 


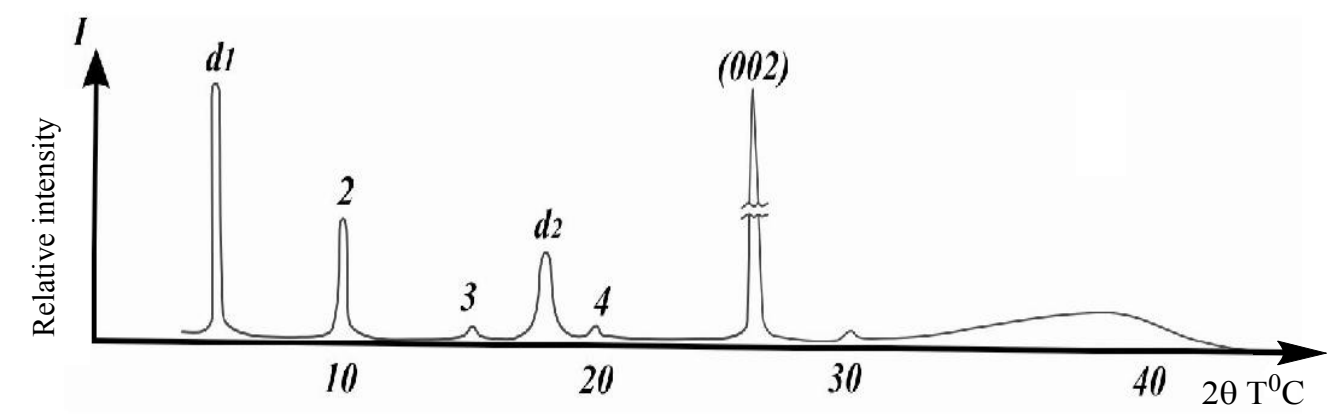

(a)

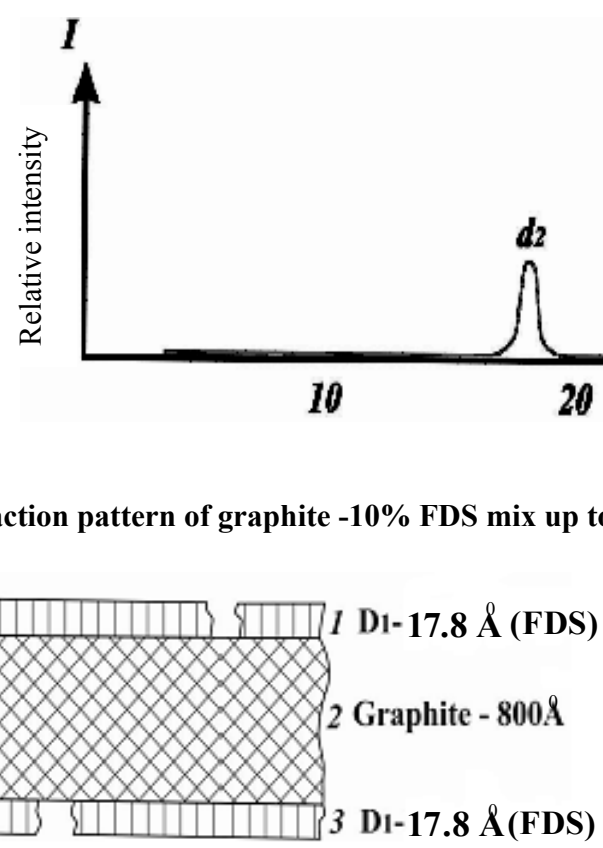

Fig. 4 Position of FDS mono-layers $\left(1,3, d_{1}=17.80 \AA\right)$ in graphite particle $(2,800 \AA)$.

the form of graphite particles covered by thin FDS film (Fig. 4). Such FDS mono layers prevent friction of graphite particles on one another and guarantee border lubrication of graphite particles. This is namely what prevents destruction of graphite particles in vibrating mill. Disappearance of orders of lines corresponding to periodicity $d_{1}$, and preservation of lines $d_{2}$ are detected by the use of diffraction patterns of mixtures containing 5\% and $10 \%$ FDS. At the FDS concentration $1 \%$ its amount is too small to show its lines on diffraction patterns. But even in this case as a result of treatment in vibrating mill or at the friction between two flat counter bodies, graphite lines (002) do not undergo any significant change, similar to those in the mixes by $5 \%$ and $10 \%$ FDS and in distinct from pure graphite.

\section{Conclusions}

Thus, particles containing a package of FDS layers, in the process of mechanical impact undergo destruction into separate mono-layers. Such destruction probably proceeds easier, since inter-laminar molecular forces of FDS of polytetrafluor ethylene structure should be very weak. These mono-layers, spread at the border of graphite crystals, provide preservation of structure of separate crystals and that of the whole mix at mechanical load, and correspondingly, low friction coefficient, both inter-crystal and at the solid surface of a mix.

Application of thus modified graphite as fillers in some polymer self-lubricating material and as additive to some plastic and liquid lubricating materials, will enable us to improve significantly their tribotechnical characteristics. 


\section{References}

[1] Marmer, E. I. 1973. Coal-graphite Materials. Moscow: Metalurgia Publishers.

[2] Chichinadze, A. V. 2003. Friction, Wear and Lubrication. Moscow: Machinostroenie Publishers.

[3] Krespan, C. G. 1972. "Synthesis and Pyrolysis of Fluorosulfates." J. Fluorine Chem. 2 (2): 173-80.
[4] Ubbelode, A. R., and Reynolds, W. N. 1968. Physical Properties of Graphite. Amsterdam: Elsevier Publishing Co. Ltd.

[5] Wunderlich, B. 1976. Physics of Micromolecules. Moscow: Mir Publishers.

[6] Kennard, O. 1972. Molecular Structures and Dimensions. Vol. A.1, Cambridge: Crystallographic Data Centre, England. 